\title{
Wolfenstein potentials for neutrinos induced by ultra-light mediators
}

\author{
Alexei Yu. Smirnov ${ }^{a, b}$ and Xun-Jie $\mathbf{X u}^{a}$ \\ ${ }^{a}$ Max-Planck-Institut für Kernphysik, \\ Postfach 103980, D-69029 Heidelberg, Germany \\ ${ }^{b}$ Abdus Salam International Centre for Theoretical Physics (ICTP), \\ I-34100 Trieste, Italy \\ E-mail: smirnov@mpi-hd.mpg.de, xunjie.xu@gmail.com
}

ABSTRACT: New physics can emerge at low energy scales, involving very light and very weakly interacting new particles. These particles can mediate interactions between neutrinos and usual matter and contribute to the Wolfenstein potential relevant for neutrino oscillations. We compute the Wolfenstein potential in the presence of ultra-light scalar and vector mediators and study the dependence of the potential on the mediator mass $m_{A}$, taking the finite size of matter distribution (Earth, Sun, supernovae) into consideration. For ultra-light mediators with $m_{A}^{-1}$ comparable to the size of the medium $(R)$, the usual $m_{A}^{-2}$ dependence of the potential is modified. In particular, when $m_{A}^{-1} \gg R$, the potential does not depend on $m_{A}$. Taking into account existing bounds on light mediators, we find that for the scalar case significant effects on neutrino propagation are not possible, while for the vector case large matter effects are allowed for $m_{A} \in\left[2 \times 10^{-17}, 4 \times 10^{-14}\right] \mathrm{eV}$ and the gauge coupling $g \sim 10^{-25}$.

KeYwords: Neutrino Physics, Solar and Atmospheric Neutrinos

ArXiv EPRINT: 1909.07505 


\section{Contents}

1 Introduction 1

2 Effects of light mediators on neutrino propagation 2

3 Effective potentials for spherically symmetric density distributions $\quad 4$

4 Phenomenology $\quad 8$

5 Conclusion

\section{Introduction}

Coherent forward scattering of neutrinos on particles of medium $\psi\left(\psi=e^{-}, n, p\right)$ generates the Wolfenstein potential $V_{W}$ [1]. Being added to the neutrino evolution equation, $V_{W}$ can significantly affect neutrino oscillations, known as the Mikheyev-Smirnov-Wolfenstein (MSW) effect [1-3]. When neutrino-matter interactions are mediated by a heavy boson with the interaction radius $m_{A}^{-1}$ (where $m_{A}$ is the mediator mass) much smaller than the object in which neutrinos propagate (or the distance over which the density varies), the Wolfenstein potential equals

$$
V_{W}=\frac{g_{\nu} g_{\psi}}{m_{A}^{2}} n_{\psi},
$$

where $n_{\psi}$ is the number density of the $\psi$ particles, $g_{\nu}$ and $g_{\psi}$ are the couplings of mediator to $\nu$ and $\psi$ respectively. The potential depends on the local number density $n_{\psi}$ while the size and shape of the object are not relevant. The medium can be considered as infinite. In the standard model (SM), the mediators are the $W$ and $Z$ bosons, satisfying the condition for (1.1). New heavy particles beyond the SM can generate via non-standard interactions additional contributions to the Wolfenstein potential with the same form as (1.1).

New neutrino interactions may be mediated by light particles as well, if the light mediators are very weakly coupled to the SM fermions. With sufficiently small values of $g_{\nu}$ and $g_{\psi}$, and correspondingly small $m_{A}$, sizable $g_{\nu} g_{\psi} / m_{A}^{2}$ can evade various bounds from processes with large momentum transfer $\left|q^{2}\right| \gg m_{A}^{2}$, because the new physics contributions in such processes are typically proportional to the small quantity $g_{\nu} g_{\psi} /\left|q^{2}\right|$ rather than $g_{\nu} g_{\psi} / m_{A}^{2}$. In contrast, the Wolfenstein potential in (1.1), can be unchanged if $m_{A}^{2}$ decreases proportionally with respect to $g_{\nu} g_{\psi}$. This, however, is restricted by the finite size of the object $R$. When $m_{A}$ becomes smaller than $1 / R$, the dependence of $V_{W}$ on $m_{A}$ in (1.1) is modified so that the matter effect turns out to be also suppressed. In this paper we will consider this dependence and its implications in details.

The matter effects due to light mediators have been studied before [4-21]. The mediators, mostly considered in the literature, are new gauge bosons of the lepton numbers 
$L_{e}-L_{\mu}, L_{\mu}-L_{\tau}$ or $L_{\tau}-L_{e}$. Long-range forces induced by these bosons can affect solar and atmospheric neutrino oscillations $[4,5]$ as well as high energy astrophysical neutrinos interacting with electrons in the Universe [15]. Various fifth force and gravitational experiments put very strong bounds on couplings of light mediators with matter but in certain ranges it is neutrino oscillation phenomena that have the best sensitivity to couplings [17]. As for scalar interactions, it is well known that the corresponding matter effect leads to corrections to the neutrino masses. Recently this possibility has been studied in ref. [18] and it is claimed that such scalar interactions can explain the discrepancy between the solar neutrino and KamLAND determinations of $\Delta m_{21}^{2}$.

In this paper, we present detailed study of the Wolfenstein potentials induced by light mediators (both vector and scalar). We compute the Wolfenstein potentials for several spherically symmetric density profiles and study dependence of the potentials on the mediator mass. Taking into account existing bounds on light mediators, we assess their relevance to neutrino experiments.

The paper is organized as follows. In section 2 we study the effects of light scalar and vector mediators on neutrino propagation, considering general matter density distributions. In section 3 we present derivation of the effective potentials for several spherically symmetric density distributions which can be applied to the Earth, the Sun and similar celestial bodies. In section 4 we consider existing bounds on light mediators and apply our results to neutrinos propagating in the Sun, the Earth and supernovae. Discussion and conclusions are presented in section 5 .

\section{Effects of light mediators on neutrino propagation}

Let us consider interactions between neutrinos $(\nu)$ and particles in matter $(\psi)$ mediated by a new light vector boson $A^{\mu}$ or scalar boson $\phi$. The relevant part of the Lagrangian reads

$$
\mathcal{L} \supset \bar{\nu} i \not \partial \nu-m_{\nu} \bar{\nu} \nu-g_{\nu} \bar{\nu} A \nu-g_{\psi} \bar{\psi} A \psi-\frac{m_{A}^{2}}{2} A^{\mu} A_{\mu}
$$

in the vector case. In the case of a scalar mediator, the last three terms in (2.1) should be replaced by

$$
\mathcal{L} \supset-y_{\nu} \bar{\nu} \phi \nu-y_{\psi} \bar{\psi} \phi \psi-\frac{m_{\phi}^{2}}{2} \phi^{2} .
$$

We assume that neutrinos are Dirac particles. For Majorana neutrinos, though the interaction forms are slightly different, the results are the same. Also we consider neutrinos of a single flavor. It can be straightforwardly generalized to the case of three-neutrino mixing.

The Lagrangian (2.1) determines the equations of motion (EOM) of $\nu$ and $A^{\mu}$ :

$$
\begin{aligned}
i \not \partial \nu-m_{\nu} \nu-g_{\nu} A \nu & =0 \\
{\left[\partial^{2}+m_{A}^{2}\right] A^{\mu}-g_{\nu} \bar{\nu} \gamma_{\mu} \nu-g_{\psi} \bar{\psi} \gamma_{\mu} \psi } & =0
\end{aligned}
$$

According to eq. (2.3), the effect of $A^{\mu}$ on neutrino propagation can be described as the displacement $i \not \partial \rightarrow i \not \partial-g_{\nu} A$, which in the momentum space corresponds to

$$
p^{\mu} \rightarrow p^{\mu}+g_{\nu} A^{\mu}
$$


where $p^{\mu}$ is the 4 -momentum of the neutrino. In particular, the neutrino energy $E$ receives the correction:

$$
E=p^{0} \rightarrow E+V, \quad V=g_{\nu} A^{0} .
$$

In the scalar case, the EOM from eq. (2.2) are

$$
\begin{aligned}
i \not \partial \nu-m_{\nu} \nu-y_{\nu} \phi \nu & =0 \\
{\left[\partial^{2}+m_{\phi}^{2}\right] \phi-y_{\nu} \bar{\nu} \nu-y_{\psi} \bar{\psi} \psi } & =0 .
\end{aligned}
$$

As follows from eq. (2.7), the effect of $\phi$ on neutrino propagation is equivalent to changing the neutrino mass:

$$
m_{\nu} \rightarrow m_{\nu}+\delta m_{\nu}, \quad \delta m_{\nu}=y_{\nu} \phi
$$

In most applications, the medium particles $\psi$ are at rest (non-relativistic), hence ${ }^{1}$

$$
\bar{\psi} \psi=n_{\psi}, \bar{\psi} \gamma^{\mu} \psi=n_{\psi}(1,0,0,0) .
$$

Since the neutrino number density is much smaller than the number density of electrons or nucleons, we take $\bar{\nu} \nu \ll \bar{\psi} \psi$ and $\bar{\nu} \gamma_{\mu} \nu \ll \bar{\psi} \gamma_{\mu} \psi$ in eqs. (2.8) and (2.4). This means that $\phi$ and $A^{\mu}$ are dominantly induced by $\psi$.

For the vector case, eq. (2.10) implies that the spatial components of $A^{\mu}$ vanish (up to gauge uncertainties):

$$
A^{\mu}=\left(A^{0}, 0,0,0\right)
$$

Furthermore, since the $\psi$ particles are at rest, $A^{0}$ has no temporal dependence $\left(\partial_{t} A^{0}=0\right)$. Therefore, eq. (2.4) becomes

$$
\left[-\nabla^{2}+m_{A}^{2}\right] A^{0}=g_{\psi} n_{\psi}
$$

Given a distribution of $n_{\psi}$, eq. (2.12) determines $A^{0}$.

All the above analyses can be straightforwardly applied to a scalar mediator. Starting from eq. (2.8), we obtain the equation similar to eq. (2.12):

$$
\left[-\nabla^{2}+m_{\phi}^{2}\right] \phi=y_{\psi} n_{\psi},
$$

and hence a similar solution.

From eqs. (2.12) and (2.13), we can see that $\phi$ and $A^{0}$ are determined by $n_{\psi}$ in the same way. However, the effects of $\phi$ and $A^{0}$ on neutrino propagation are very different.

In the neutrino evolution equation $\phi$ gives correction to the mass and therefore appears as $\left(m_{\nu}+\delta m_{\nu}\right)^{2} / 2 E$. Approximately(for $\left.\delta m_{\nu} \ll m_{\nu}\right)$, this corresponds to adding

$$
V_{S} \approx \frac{m_{\nu}}{E} \delta m_{\nu}=\frac{m_{\nu}}{E} y_{\nu} \phi
$$

to $\left(m_{\nu}\right)^{2} / 2 E$. In comparison, $A^{0}$ appears in the equation as an addition of $V=g_{\nu} A^{0}$ to $m_{\nu}^{2} / 2 E$. Thus, the scalar matter effect enters the flavor evolution equation with the

\footnotetext{
${ }^{1}$ Recall that the physical meaning of $\bar{\psi} \gamma^{\mu} \psi$ is the electric current density and $\bar{\psi} \gamma^{0} \psi=\psi^{\dagger} \psi$ is the electron number density [22]. For $\psi$ at rest, $\bar{\psi} \psi=\bar{\psi} \gamma^{0} \psi$ is identical to the electron number density.
} 
additional suppression factor $m_{\nu} / E$. This factor is due to chiral suppression. Since the helicity is conserved in neutrino oscillations, the chirality-flipping terms in the Lagrangian, such as mass terms or scalar interactions, have to change it twice, which is the reason that $m_{\nu}^{2}$ appears instead of $m_{\nu}$ in neutrino oscillations. Because the scalar interaction flips the chirality, another flip is needed which is given by $m_{\nu} / 2 E$. In other words, only $m_{\nu} / 2 E$ fraction of the chirality-flipped state contains the original helicity. This means that, to obtain effects of the same size, $y_{\nu} \phi$ should be $\frac{E}{m_{\nu}}$ times larger than $g_{\nu} A^{0}$. That is, $y_{\nu} y_{\psi} / m_{\phi}^{2}$ should be $\frac{E}{m_{\nu}}$ times larger than $g_{\nu} g_{\psi} / m_{A}^{2}$. This strongly affects the relevance of the scalar case to the oscillation phenomenology.

In the case of pseudo-scalar and axial-vector mediators, the corresponding fields are produced by interactions with $\bar{\psi} \gamma^{5} \psi$ and $\bar{\psi} \gamma^{\mu} \gamma^{5} \psi$. In unpolarized medium, these two quantities vanish due to $\gamma^{5}$. For polarized medium, the pseudo-scalar and axial-vector potentials decrease as $r^{-3}$, being dipole effects (see, e.g., ref. [23]). Therefore these potentials are small compared to scalar and vector potentials.

\section{Effective potentials for spherically symmetric density distributions}

In what follows, we consider the vector case, while for the scalar case, the results can be obtained immediately with the substitutions: $A^{0} \rightarrow \phi, m_{A} \rightarrow m_{\phi}, g_{\nu} \rightarrow y_{\nu}$, and $g_{\psi} \rightarrow y_{\psi}$.

In many applications (e.g., the Earth, the Sun), the matter density distribution is, to a good approximation, spherically symmetric. The spherical symmetry allows us to reduce eq. (2.12) to a radial differential equation:

$$
\left[\frac{\partial^{2}}{\partial r^{2}}+\frac{2 \partial}{r \partial r}-m_{A}^{2}\right] A^{0}(r)=-g_{\psi} n_{\psi}(r)
$$

For any given profile $n_{\psi}(r)$, eq. (3.1) can be solved by a standard method known as variation of parameters, which gives

$$
A^{0}(r)=\frac{g_{\psi}}{m_{A} r}\left[e^{-m_{A} r} \int_{0}^{r} x n_{\psi}(x) \sinh \left(m_{A} x\right) d x+\sinh \left(m_{A} r\right) \int_{r}^{\infty} x n_{\psi}(x) e^{-m_{A} x} d x\right] .
$$

The above computations of potentials are essentially classical. Therefore for an arbitrary density distribution $n_{\psi}(\boldsymbol{r})$ (not necessarily spherically symmetric), $A^{0}$ can be found by performing summation (integration) of the Yukawa potentials produced by individual particles [see also eq. (3.8)]:

$$
A^{0}(\boldsymbol{r})=-\frac{g_{\psi}}{4 \pi} \int n_{\psi}(\tilde{\boldsymbol{r}}) \frac{e^{-m_{A}|\boldsymbol{r}-\tilde{\boldsymbol{r}}|}}{|\boldsymbol{r}-\tilde{\boldsymbol{r}}|} d^{3} \tilde{\boldsymbol{r}}
$$

For the spherically symmetric case, one can integrate over angular variables in eq. (3.3), which also leads to eq. (3.2).

Using eq. (3.2) we compute the Wolfenstein potentials for several matter density profiles, which can be used to approximately describe the density distributions of the Earth, the Sun, and supernovae. Some interesting limits will be discussed. 
Constant density within a sphere. For a constant density distribution within a sphere of radius $R$ :

$$
n_{\psi}(r)= \begin{cases}0 & (\text { for } r>R) \\ n_{\psi} & (\text { for } r \leq R)\end{cases}
$$

we find from (3.2)

$$
A^{0}(r)=\frac{g_{\psi} n_{\psi}}{m_{A}^{2}} F(r)
$$

where

$$
F(r)=\left\{\begin{array}{ll}
1-\frac{m_{A} R+1}{m_{A} r} e^{-m_{A} R} \sinh \left(m_{A} r\right) & (r \leq R) \\
\frac{e^{-m_{A} r}}{m_{A} r}\left[m_{A} R \cosh \left(m_{A} R\right)-\sinh \left(m_{A} R\right)\right] & (r>R)
\end{array},\right.
$$

describes deviation from the infinite medium potential. Then according to eq. (2.6), the effective neutrino potential produced by $A^{0}$ equals

$$
V(r)=g_{\nu} A^{0}(r)=\frac{g_{\nu} g_{\psi} n_{\psi}}{m_{A}^{2}} F(r) .
$$

Several important limits are in order.

- $R \rightarrow 0$. We fix the number of particles $\psi, N_{\psi} \equiv \frac{4}{3} \pi R^{3} n_{\psi}$, within the sphere when taking $R \rightarrow 0$. In this limit $n_{\psi}(r) \rightarrow N_{\psi} \delta(r)$, eqs. (3.6) and (3.7) give

$$
V(r)=\frac{g_{\nu} g_{\psi}}{m_{A}^{2}} \frac{e^{-m_{A} r}}{r} n_{\psi} \frac{R^{3}}{3}=\frac{g_{\nu} g_{\psi}}{m_{A}^{2}} N_{\psi} \frac{e^{-m_{A} r}}{4 \pi r}
$$

which, for $N_{\psi}=1$, reproduces the Yukawa potential of a single $\psi$ particle.

- $m_{A} \rightarrow 0$. In the limit of massless mediator, eqs. (3.6) and (3.7) lead to

$$
V(r)=g_{\nu} g_{\psi} n_{\psi} \times \begin{cases}\frac{3 R^{2}-r^{2}}{6} & (r \leq R) \\ \frac{R^{3}}{3 r} & (r>R)\end{cases}
$$

which coincides with the $r$ dependence of the Coulomb potential of a charged sphere. In this limit, the potential for $r>R$ is determined by the total particle number $N_{\psi}$ inside the sphere, independently of the radial distribution. If $m_{A}$ is nonzero but small, the first order correction in $m_{A}$ to the potential equals (for both $r \leq R$ and $r>R)$

$$
\delta V(r)=-g_{\nu} g_{\psi} n_{\psi} \frac{m_{A}}{3} R^{3} .
$$

- $m_{A} \rightarrow \infty$. In this limit, eqs. (3.6) and (3.7) give

$$
V(r)=\left\{\begin{array}{ll}
\frac{g_{\nu} g_{\psi} n_{\psi}}{m_{A}^{2}} & (r \leq R) \\
0 & (r>R)
\end{array} .\right.
$$

Inside medium, the potential is a constant and outside it vanishes. This reproduces the standard Wolfenstein potential for infinite medium. 

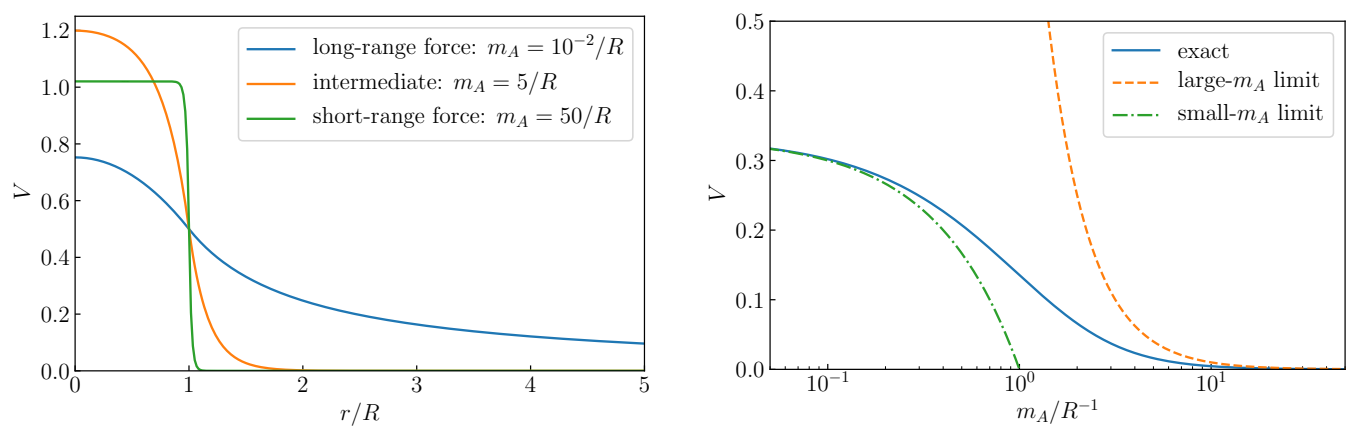

Figure 1. The effective potentials $V$ (in arbitrary unit) produced by a sphere of radius $R$ with constant matter density. Left panel: dependence of $V$ on the distance $r$ for different values of the mediator mass $m_{A}$. Values of $g_{\nu} g_{\psi} n_{\psi}$ are chosen in such a way that $V(r=R)=1 / 2$ for all values of $m_{A}$. Right panel: comparison of $V\left(m_{A}\right)$ in the large and small $m_{A}$ limits with the exact result, computed using eqs. (3.5)-(3.7) and (3.9)-(3.11) at the surface of the sphere $(r=R)$.

- $|R-r| \ll m_{A}^{-1} \ll R$. The limit means that the radius of interaction is much smaller than $R$ but much larger than the depth of the trajectory $R-r$. The absolute sign "I |" indicates that this limit applies not only to underground neutrino trajectories but also to above-the-surface neutrino beams. The limit can be realized for reactor and accelerator experiments. In this limit, we obtain

$$
V(r)=\frac{1}{2} \frac{g_{\nu} g_{\psi} n_{\psi}}{m_{A}^{2}}
$$

It differs from the standard Wolfenstein potential by the additional factor of $1 / 2$, which reflects that only half of the space produces the potential.

In the left panel of figure 1, we show the dependence of $V$ on $r$ for different values of $m_{A}$ according to (3.7) and (3.6). For $m_{A} \gg R^{-1}$ (green curve) the $r$-dependence is close to that of the standard Wolfenstein potential, which is essentially a step function. Near the surface $(r \approx R)$ the short-range potential (green curve) is roughly half the standard Wolfenstein potential (the plateau of this curve), as expected from eq. (3.12). When $m_{A}$ decreases, it becomes smoother (yellow line). For very small $m_{A}$, the long-range force leads to a Column-like potential (blue curve).

In the right panel of figure 1, we show the dependence of $V$ at $r=R$ on $m_{A}$ according to the exact formula (blue solid curve) as well as the small- and large- $m_{A}$ limits in eqs. (3.9)-(3.11). The inverse-square dependence of the standard Wolfenstein potential on $m_{A}$ deviates significantly from the exact dependence for small values of $m_{A}$.

Multi-layer density profile. A multi-layer density profile with constant densities within layers is a good approximation for the Earth density distribution. Using result (3.6) for constant density spheres one can obtain results for the Earth. For instance, for two layers (a simplified mantle-core profile) with constant average densities $n_{\psi}^{M}$ and $n_{\psi}^{C}$ and radii $R^{M}$ and $R^{C}$ respectively, the Wolfenstein potential in the mantle is

$$
V(r)=\frac{g_{\nu} g_{\psi}}{m_{A}^{2}}\left[n_{\psi}^{M} F_{r<R}\left(r, R^{M}\right)+\left(n_{\psi}^{C}-n_{\psi}^{M}\right) F_{r>R}\left(r, R^{C}\right)\right],
$$


and in the core is

$$
V(r)=\frac{g_{\nu} g_{\psi}}{m_{A}^{2}}\left[n_{\psi}^{M} F_{r<R}\left(r, R^{M}\right)+\left(n_{\psi}^{C}-n_{\psi}^{M}\right) F_{r<R}\left(r, R^{C}\right)\right],
$$

where $F_{r<R}$ and $F_{r>R}$ are given by the lower and the upper lines of eq. (3.6). For more realistic profiles with many layers, the generalization is straightforward.

Exponential density distribution. Exact analytic results can be obtained for an exponential density distribution,

$$
n_{\psi}(r)=n_{\psi}(0) e^{-r \kappa} .
$$

This distribution can be used to partially describe the matter density of the Sun and supernovae. Computing the integral in (3.2) gives

$$
V(r)=\frac{g_{\nu} g_{\psi} n_{\psi}(r)}{m_{A}^{2}-\kappa^{2}}\left[1+\frac{2 \kappa}{m_{A}^{2}-\kappa^{2}} \frac{1}{r}\left(e^{\left(\kappa-m_{A}\right) r}-1\right)\right] .
$$

Several important limits are in order.

- $\kappa \rightarrow 0$. This limit corresponds to a constant density distribution. In this limit, eq. (3.16) reduces to the standard Wolfenstein potential of infinite medium, see eq. (3.11) with $n_{\psi}=n_{\psi}(0)$.

- $m_{A} \ll \kappa$. In this limit, the radius of force is much larger than the scale of density change. From eq. (3.16), we obtain

$$
V(r)=-\frac{g_{\nu} g_{\psi} n_{\psi}(r)}{\kappa^{2}}\left[1-\frac{2}{\kappa r}\left(e^{\kappa r}-1\right)\right],
$$

which does not depend on $m_{A}$. For $r \gg \kappa^{-1}$, eq. (3.17) reduces to

$$
V(r)=\frac{g_{\nu} g_{\psi} n_{\psi}(0)}{\kappa^{2}} \frac{2}{\kappa r},
$$

that is, the potential at large $r$ decreases as $1 / r$, i.e., slower than the matter density $(\propto$ $\left.e^{-\kappa r}\right)$ decreases. This can produce interesting phenomena such as new level crossing for solar neutrinos [24].

- $m_{A} \approx \kappa$. In this limit, eq. (3.16) gives

$$
V(r)=\frac{g_{\nu} g_{\psi} n_{\psi}(r)}{4 \kappa^{2}}(1+\kappa r) .
$$

Exponential density distribution with a cut-off. The density profile of the Sun can be described more accurately than in (3.15) by an exponential distribution with a cut-off at the solar radius:

$$
n_{\psi}(r)=n_{\psi}(0)\left\{\begin{array}{ll}
e^{-r \kappa} & (r \leq R) \\
0 & (r>R)
\end{array}\right. \text {. }
$$


After straightforward calculations, we obtain the potential:

$$
V(r)=\frac{g_{\nu} g_{\psi} n_{\psi}(0)}{m_{A} r} \times \begin{cases}K_{\mathrm{in}} & (r \leq R) \\ K_{\mathrm{out}} & (r>R)\end{cases}
$$

with $K_{\text {in }}$ and $K_{\text {out }}$ given by

$$
\begin{gathered}
K_{\text {in }} \equiv \kappa m_{A} e^{-r\left(\kappa+m_{A}\right)} \frac{e^{m_{A} r}\left(m_{A}^{2} r \kappa^{-1}-\kappa r-2\right)+2 e^{\kappa r}}{\left(m_{A}^{2}-\kappa^{2}\right)^{2}} \\
-\frac{\sinh \left(m_{A} r\right) e^{-R\left(\kappa+m_{A}\right)}\left(m_{A} R+\kappa R+1\right)}{\left(m_{A}+\kappa\right)^{2}} \\
K_{\text {out }} \equiv e^{-m_{A} r-\kappa R} \sinh \left(m_{A} R\right) \frac{m_{A}^{2}(\kappa R-1)-\kappa^{2}(\kappa R+1)}{\left(m_{A}^{2}-\kappa^{2}\right)^{2}} \\
+e^{-m_{A} r-\kappa R} \cosh \left(m_{A} R\right) \frac{m_{A}^{3} R-\kappa^{2} R m_{A}-2 \kappa m_{A}}{\left(m_{A}^{2}-\kappa^{2}\right)^{2}}+e^{-m_{A} r} \frac{2 \kappa m_{A}}{\left(m_{A}^{2}-\kappa^{2}\right)^{2}} .
\end{gathered}
$$

All the above results can be applied to the scalar case with the simple substitution: $A^{0} \rightarrow \phi, m_{A} \rightarrow m_{\phi}, g_{\nu} \rightarrow y_{\nu}$, and $g_{\psi} \rightarrow y_{\psi}$.

\section{Phenomenology}

Let us consider experimental bounds on the couplings and masses of light mediators and check whether light scalar and vector mediators, that satisfy these bounds, can produce observable matter effects in neutrino oscillations. For brevity we introduce the couplings $g \equiv \sqrt{g_{\nu} g_{\psi}}$ and $y \equiv \sqrt{y_{\nu} y_{\psi}}$.

For $m_{A, \phi} \gtrsim \mathcal{O}\left(10^{2}\right) \mathrm{keV}, g^{2} / m_{A}^{2} \gtrsim G_{F}$ and $y^{2} / m_{\phi}^{2} \gtrsim G_{F}$ have been excluded by numerous laboratory experiments including the elastic neutrino-electron scattering [25-27], neutrino-nucleus scattering [28-30], fixed target experiments [31-33], collider searches [34, 35], etc. For $1 \mathrm{eV} \lesssim m_{A, \phi} \lesssim 10^{2} \mathrm{keV}$, the astrophysical observations provide much stronger constraints. For instance, the expected amount of energy loss via neutrinos in the Sun and globular clusters excludes $g$ and $y$ down to $10^{-14}$, corresponding to an upper bound of $g^{2} / m_{A}^{2}$ or $y^{2} / m_{\phi}^{2}$ above $10^{-5} G_{F}$ [36].

Below $1 \mathrm{eV}$, the constraints mainly come from searches of fifth forces and precision tests of gravity $[37,38]$. These constraints are only applicable to $g_{\psi}$ or $y_{\psi}$. To obtain the bounds on $\sqrt{g_{\nu} g_{\psi}}$ or $\sqrt{y_{\nu} y_{\psi}}$, we can use the cosmological bounds on neutrino self-interactions, $g_{\nu}^{2} / m_{A}^{2}$ or $y_{\nu}^{2} / m_{\phi}^{2} \lesssim(3 \mathrm{MeV})^{-2}$ [39]. In addition, for certain ranges of $m_{A, \phi}$, the black hole super-radiance provides robust constraints $[15,40]$ which are independent of the couplings. These constraints are combined and presented in figure 2 .

Notice that these constrains for the mass of mediators below $1 \mathrm{eV}$ (shown in figure 2) are based on tests of gravity and cosmological observations, and therefore flavor independent. Searches for the fifth force and precision tests of gravity are sensitive to the couplings of mediators with matter $g_{\psi}\left(y_{\psi}\right)$, while the bounds on neutrino couplings $g_{\nu}\left(y_{\nu}\right)$ are based 

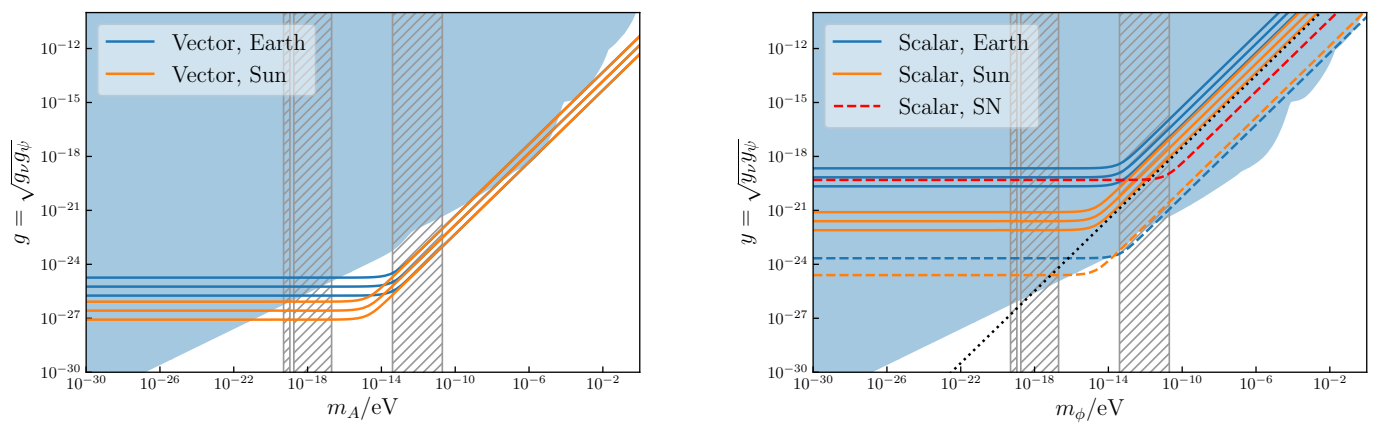

Figure 2. The regions of masses and couplings of ultra-light vector (left) and scalar (right) mediators in which significant effects are produced by the matter of the Earth at its surface (blue lines) and by matter of the Sun in its center (orange lines). The blue shaded regions are excluded by the combination of various observations (explained in the text). The grey hatched regions are excluded by black hole super-radiance. Left panel: lines of equal potentials generated by vector mediator. From down to up: $V / V_{\mathrm{SM}}=\left\{10^{-2}, 10^{-1}, 1\right\}$. Right panel: lines of equal corrections to the neutrino mass generated by scalar mediator. From down to up: $\delta m_{\nu} / \mathrm{eV}=\left\{10^{-3}, 10^{-2}, 10^{-1}\right\}$. For comparison, we plot two dashed lines with $\delta m_{\nu}=10^{-11} \mathrm{eV}$ for the Earth and $\delta m_{\nu}=10^{-8} \mathrm{eV}$ for the Sun which have parts below the excluded regions. Red dashed line corresponds to $\delta m_{\nu}=5$ $\mathrm{MeV}$ generated by the core of supernovae. The region above this line is excluded (see text).

on the effect of neutrino free streaming on CMB, which are independent of neutrino flavors. Above $1 \mathrm{eV}$, some of the mentioned constraints, e.g., from neutrino scattering, do depend on flavors. However, studies in this region of masses show that new interactions cannot be much larger than the SM interactions. Therefore above $1 \mathrm{eV}$ the scalar case remains excluded, whereas the vector case still has viable parameter space, as has been widely discussed in the literature.

Now let us determine the required values of $g$ or $y$ to generate significant matter effects. The latter can be quantified by

$$
\frac{V}{V_{\mathrm{SM}}}=\frac{g^{2}}{m_{A}^{2} \sqrt{2} G_{F}} F\left(r, m_{A}^{2}\right), \quad \delta m_{\nu}=\frac{y^{2}}{m_{\phi}^{2}} n_{\psi}(r) F\left(r, m_{\phi}^{2}\right),
$$

where $V_{\mathrm{SM}} \equiv \sqrt{2} G_{F} n_{\psi}$, and $F$ presented in eqs. (3.6) describes the deviation from the standard Wolfenstein potential. According to eq. (4.1), for given values of $\frac{V}{V_{\mathrm{SM}}}$ and $\delta m_{\nu}$, $g^{2}$ and $y^{2}$ are determined by:

$$
g^{2}=\frac{V}{V_{\mathrm{SM}}} m_{A}^{2} \sqrt{2} G_{F} F^{-1}\left(r, m_{A}^{2}\right), \quad y^{2}=\delta m_{\nu} m_{\phi}^{2}\left[n_{\psi(r)} F\left(r, m_{\phi}^{2}\right)\right]^{-1} .
$$

In figure 2, we plot the dependence $g=g\left(m_{A}\right)$ and $y=y\left(m_{\phi}\right)$ from eq. (4.2) for $V / V_{\mathrm{SM}} \in$ $\left\{10^{-2}, 10^{-1}, 1\right\}$ and $\delta m_{\nu} \in\left\{10^{-3}, 10^{-2}, 10^{-1}\right\} \mathrm{eV}$ correspondingly. The curves determine the bands of strong (observable) matter effects in the mediator parameter space. The values of $F$ have been computed numerically for the center of the Sun $(r=0)$ using the solar density distributions from [41], and for the surface of the Earth $\left(r=R_{\oplus}\right)$ using the density distribution from [42]. The value of $F$ has been computed numerically using the density 
distributions for the Sun from [41] and for the Earth from [42]. The value of $r$ is set to zero for the Sun, and to the Earth radius for the Earth.

The dependence $g=g\left(m_{A}\right)$ and $y=y\left(m_{\phi}\right)$ can be understood from our analytic results. For large $m_{A}$ (short range forces), $F \approx 1$. According to eq. (4.2), we have $g \propto m_{A}^{2}$ for $m_{A} \ll 1 / R$, where $R$ is the radius of the object. For very small $m_{A}, F$ should be proportional to $m_{A}^{2}$ so that $g$ is independent of $m_{A}$. In the intermediate range, $m_{A} \sim 1 / R$, the dependence is more complicated.

According to figure 2, the solid curves turn at the values of mediator masses $2.8 \times 10^{-16} \mathrm{eV}$ for the Sun, and $3.1 \times 10^{-14} \mathrm{eV}$ for the Earth that are determined by inverse of the solar radius $R_{\odot}=7.0 \times 10^{5} \mathrm{~km}$ and the Earth radius $R_{\oplus} \approx 6.4 \times 10^{3} \mathrm{~km}$ correspondingly. Below these masses at $m_{A}, m_{\phi}<10^{-16} \mathrm{eV}$ (for the Sun) and $m_{A}, m_{\phi}<10^{-14} \mathrm{eV}$ (for the Earth) the curve becomes horizontal, i.e. dependence on the mediator masses disappears. This corresponds essentially to the Coulomb-like potentials generated by massless mediators.

According to figure 2 (left) there are several $m_{A^{-}} g$ regions of observable matter effects which are generated by the vector mediator and allowed by the present bounds. In the long-range forces domain they include

$$
m_{A}=\left(10^{-21}-10^{-19}\right) \mathrm{eV}, \quad g=\left(10^{-27}-10^{-26}\right),
$$

for neutrinos in the Sun, and

$$
m_{A}=\left(10^{-21}-10^{-19}\right) \mathrm{eV}, \quad g=\left(10^{-27}-10^{-26}\right),
$$

both for neutrinos in the Sun and in the Earth. The unexcluded regions in the short range forces domain are the same for the neutrinos in the Sun and the Earth:

$$
\begin{array}{ll}
m_{A}=\left(10^{-11}-10^{-6}\right) \mathrm{eV}, & g=\left(10^{-24}-10^{-19}\right), \\
m_{A}>10^{-4} \mathrm{eV}, & g>10^{-17} .
\end{array}
$$

This could motivate further phenomenological studies.

As follows from figure 2 (right panel) the scalar mediators cannot generate significant matter effects, in contrast to the claim in ref. [18]. For comparison, a black dotted line corresponds to $\delta m_{\nu}=0.001 \mathrm{eV}$ generated in the infinite size medium with the same density as in the center of the Sun. (For large $m_{\phi}$ it coincides with the corresponding line for the Sun.) In this case, significant matter effects would be possible for $m_{\phi} \lesssim 10^{-20} \mathrm{eV}$ ). However, this possibility is excluded when $F(r)$ (the correction) due to the finite size of medium is taken into account.

One may be interested in the allowed values of scalar matter effect. So, we plot two dashed curves that correspond to values of corrections $\delta m_{\nu}=10^{-11} \mathrm{eV}$ for the Earth and $\delta m_{\nu}=10^{-8} \mathrm{eV}$ which have small sections below the excluded region. These values are far beyond the precision of any realistic experiments.

Finally, strong constraints on the scalar mediator parameters can be obtained from supernova neutrinos, which have not been considered in the literature. Let us estimate the 
effect in the core of a supernova with a typical radius of $R_{\mathrm{SN}}=20 \sim 30 \mathrm{~km}$ and a density of $n_{\psi}^{\mathrm{SN}}=10^{13} \sim 10^{14} \mathrm{~g} / \mathrm{cm}^{3}$. For relatively heavy scalar mediators with $m_{\phi}>1 / R_{\mathrm{SN}}$, the mass correction

$$
\delta m_{\nu}^{\mathrm{SN}} \sim \frac{n_{\psi}^{\mathrm{SN}}}{n_{\psi}^{\mathrm{earth}}} \delta m_{\nu}^{\mathrm{earth}},
$$

is about 13 orders of magnitude larger than the correction in the Earth $\delta m_{\nu}^{\text {earth }}$. For $\delta m_{\nu}^{\text {earth }}=0.01 \mathrm{eV}$, we obtain $\delta m_{\nu}^{\mathrm{SN}} \sim 100 \mathrm{GeV}$, which is certainly excluded since in this case neutrinos can not even be produced in supernovae. For very light mediators with $m_{\phi}<1 / R_{\mathrm{SN}}$, eq. (4.7) is modified to

$$
\delta m_{\nu}^{\mathrm{SN}} \sim \frac{n_{\psi}^{\mathrm{SN}}}{n_{\psi}^{\text {earth }}}\left(\frac{R^{\mathrm{SN}}}{R^{\mathrm{earth}}}\right)^{2} \delta m_{\nu}^{\mathrm{earth}}
$$

Taking $\delta m_{\nu}^{\text {earth }}=0.01 \mathrm{eV}$, we obtain $\delta m_{\nu}^{\mathrm{SN}}=5 \mathrm{MeV}$ and correspondingly, $y=10^{-20}$ (see figure 2). Since neutrino masses $m_{\nu}>5 \mathrm{MeV}$ would strongly affect production of neutrinos and their energy spectrum, values of couplings $y>10^{-20}$ can be excluded. More detailed analyses will be given elsewhere [24].

\section{Conclusion}

We studied the effects of new neutrino-matter interactions mediated by ultra-light scalar and vector bosons on neutrino propagation, taking into account the finite size and density distribution of the medium. We compute the Wolfenstein potentials explicitly for several spherically symmetric density profiles which can be approximately applied to the Earth and the Sun.

The Wolfenstein potentials induced by ultra-light mediators have very different dependence on the mediator masses $\left(m_{A}\right)$ from the standard case. In infinite medium, due to the $1 / m_{A}^{2}$ dependence in eq. (1.1), the Wolfenstein potentials can be enhanced by reducing the mediator masses. In finite medium of size $R$, when $m_{A}$ decreases down to the region where $m_{A}^{-1}$ becomes comparable or larger than $R$, the $1 / m_{A}^{2}$ dependence is modified. In this case the matter effect is determined by the geometry (including the size) and density distribution of the object, rather than the local density. In particular, the potential can extend outside of the medium. For $m_{A} \ll R$, the potential does not depend on $m_{A}$.

With correct expressions for the Wolfenstein potentials and existing bounds on light mediators, we find that scalar mediators cannot produce observable effects in realistic experiments, which implies that the scenario considered in ref. [18] is not viable. Vector mediators, however, can produce significant matter effect, in particular, within the parameter space $m_{A} \in\left[2 \times 10^{-17}, 4 \times 10^{-14}\right] \mathrm{eV}$ and $g \sim 10^{-25}$.

Open Access. This article is distributed under the terms of the Creative Commons Attribution License (CC-BY 4.0), which permits any use, distribution and reproduction in any medium, provided the original author(s) and source are credited. 


\section{References}

[1] L. Wolfenstein, Neutrino Oscillations in Matter, Phys. Rev. D 17 (1978) 2369 [InSPIRE].

[2] S.P. Mikheyev and A. Yu. Smirnov, Resonance Amplification of Oscillations in Matter and Spectroscopy of Solar Neutrinos, Sov. J. Nucl. Phys. 42 (1985) 913 [inSPIRE].

[3] S.P. Mikheev and A. Yu. Smirnov, Resonant amplification of neutrino oscillations in matter and solar neutrino spectroscopy, Nuovo Cim. C 9 (1986) 17 [INSPIRE].

[4] A.S. Joshipura and S. Mohanty, Constraints on flavor dependent long range forces from atmospheric neutrino observations at Super-Kamiokande, Phys. Lett. B 584 (2004) 103 [hep-ph/0310210] [INSPIRE].

[5] J.A. Grifols and E. Masso, Neutrino oscillations in the sun probe long range leptonic forces, Phys. Lett. B 579 (2004) 123 [hep-ph/0311141] [INSPIRE].

[6] A. Bandyopadhyay, A. Dighe and A.S. Joshipura, Constraints on flavor-dependent long range forces from solar neutrinos and KamLAND, Phys. Rev. D 75 (2007) 093005 [hep-ph/0610263] [INSPIRE].

[7] M.C. Gonzalez-Garcia, P.C. de Holanda, E. Masso and R. Zukanovich Funchal, Probing long-range leptonic forces with solar and reactor neutrinos, JCAP 01 (2007) 005 [hep-ph/0609094] [INSPIRE].

[8] A.E. Nelson and J. Walsh, Short Baseline Neutrino Oscillations and a New Light Gauge Boson, Phys. Rev. D 77 (2008) 033001 [arXiv:0711.1363] [INSPIRE].

[9] M.C. Gonzalez-Garcia, P.C. de Holanda and R. Zukanovich Funchal, Constraints from Solar and Reactor Neutrinos on Unparticle Long-Range Forces, JCAP 06 (2008) 019 [arXiv:0803.1180] [INSPIRE].

[10] A. Samanta, Long-range Forces: Atmospheric Neutrino Oscillation at a magnetized Detector, JCAP 09 (2011) 010 [arXiv: 1001.5344] [INSPIRE].

[11] J. Heeck and W. Rodejohann, Gauged $L_{\mu}-L_{\tau}$ and different Muon Neutrino and Anti-Neutrino Oscillations: MINOS and beyond, J. Phys. G 38 (2011) 085005 [arXiv:1007.2655] [INSPIRE].

[12] H. Davoudiasl, H.-S. Lee and W.J. Marciano, Long-Range Lepton Flavor Interactions and Neutrino Oscillations, Phys. Rev. D 84 (2011) 013009 [arXiv:1102.5352] [INSPIRE].

[13] H.-S. Lee, Long-range interaction effects on neutrino oscillation, J. Phys. Conf. Ser. 408 (2013) 012032 [arXiv:1110.1335] [INSPIRE].

[14] S.S. Chatterjee, A. Dasgupta and S.K. Agarwalla, Exploring Flavor-Dependent Long-Range Forces in Long-Baseline Neutrino Oscillation Experiments, JHEP 12 (2015) 167 [arXiv: 1509.03517] [INSPIRE].

[15] M. Bustamante and S.K. Agarwalla, Universe's Worth of Electrons to Probe Long-Range Interactions of High-Energy Astrophysical Neutrinos, Phys. Rev. Lett. 122 (2019) 061103 [arXiv: 1808.02042] [INSPIRE].

[16] A. Khatun, T. Thakore and S. Kumar Agarwalla, Can INO be Sensitive to Flavor-Dependent Long-Range Forces?, JHEP 04 (2018) 023 [arXiv: 1801.00949] [INSPIRE].

[17] M.B. Wise and Y. Zhang, Lepton Flavorful Fifth Force and Depth-dependent Neutrino Matter Interactions, JHEP 06 (2018) 053 [arXiv: 1803.00591] [INSPIRE]. 
[18] S.-F. Ge and S.J. Parke, Scalar Nonstandard Interactions in Neutrino Oscillation, Phys. Rev. Lett. 122 (2019) 211801 [arXiv: 1812.08376] [INSPIRE].

[19] G. Krnjaic, P.A.N. Machado and L. Necib, Distorted neutrino oscillations from time varying cosmic fields, Phys. Rev. D 97 (2018) 075017 [arXiv:1705.06740] [INSPIRE].

[20] A. Berlin, Neutrino Oscillations as a Probe of Light Scalar Dark Matter, Phys. Rev. Lett. 117 (2016) 231801 [arXiv:1608.01307] [InSPIRE].

[21] V. Brdar, J. Kopp, J. Liu, P. Prass and X.-P. Wang, Fuzzy dark matter and nonstandard neutrino interactions, Phys. Rev. D 97 (2018) 043001 [arXiv:1705.09455] [INSPIRE].

[22] M. Peskin and D. Schroeder, An Introduction to quantum field theory, Addison-Wesley, New York U.S.A. (1995).

[23] Y. Chikashige, R.N. Mohapatra and R.D. Peccei, Are There Real Goldstone Bosons Associated with Broken Lepton Number?, Phys. Lett. B 98 (1981) 265 [INSPIRE].

[24] A.Y. Smirnov and X.-J. Xu, work in preparation.

[25] W. Rodejohann, X.-J. Xu and C.E. Yaguna, Distinguishing between Dirac and Majorana neutrinos in the presence of general interactions, JHEP 05 (2017) 024 [arXiv:1702.05721] [INSPIRE].

[26] M. Lindner, F.S. Queiroz, W. Rodejohann and X.-J. Xu, Neutrino-electron scattering: general constraints on $Z^{\prime}$ and dark photon models, JHEP 05 (2018) 098 [arXiv:1803.00060] [INSPIRE].

[27] G. Arcadi, M. Lindner, J. Martins and F.S. Queiroz, New Physics Probes: Atomic Parity Violation, Polarized Electron Scattering and Neutrino-Nucleus Coherent Scattering, arXiv: 1906.04755 [INSPIRE].

[28] M. Lindner, W. Rodejohann and X.-J. Xu, Coherent Neutrino-Nucleus Scattering and new Neutrino Interactions, JHEP 03 (2017) 097 [arXiv: 1612.04150] [INSPIRE].

[29] Y. Farzan, M. Lindner, W. Rodejohann and X.-J. Xu, Probing neutrino coupling to a light scalar with coherent neutrino scattering, JHEP 05 (2018) 066 [arXiv: 1802.05171] [INSPIRE].

[30] V. Brdar, W. Rodejohann and X.-J. Xu, Producing a new Fermion in Coherent Elastic Neutrino-Nucleus Scattering: from Neutrino Mass to Dark Matter, JHEP 12 (2018) 024 [arXiv: 1810.03626] [INSPIRE].

[31] J.D. Bjorken, R. Essig, P. Schuster and N. Toro, New Fixed-Target Experiments to Search for Dark Gauge Forces, Phys. Rev. D 80 (2009) 075018 [arXiv:0906.0580] [INSPIRE].

[32] B. Batell, M. Pospelov and A. Ritz, Exploring Portals to a Hidden Sector Through Fixed Targets, Phys. Rev. D 80 (2009) 095024 [arXiv: 0906.5614] [InSPIRE].

[33] R. Essig, R. Harnik, J. Kaplan and N. Toro, Discovering New Light States at Neutrino Experiments, Phys. Rev. D 82 (2010) 113008 [arXiv:1008.0636] [INSPIRE].

[34] BABAR collaboration, Search for a Dark Photon in $e^{+} e^{-}$Collisions at BaBar, Phys. Rev. Lett. 113 (2014) 201801 [arXiv: 1406 .2980] [INSPIRE].

[35] BaBAR collaboration, Search for a muonic dark force at BABAR, Phys. Rev. D 94 (2016) 011102 [arXiv: 1606.03501] [INSPIRE].

[36] R. Harnik, J. Kopp and P.A.N. Machado, Exploring nu Signals in Dark Matter Detectors, JCAP 07 (2012) 026 [arXiv: 1202.6073] [INSPIRE]. 
[37] E.G. Adelberger, B.R. Heckel, S.A. Hoedl, C.D. Hoyle, D.J. Kapner and A. Upadhye, Particle Physics Implications of a Recent Test of the Gravitational Inverse Sqaure Law, Phys. Rev. Lett. 98 (2007) 131104 [hep-ph/0611223] [INSPIRE].

[38] S. Schlamminger, K.Y. Choi, T.A. Wagner, J.H. Gundlach and E.G. Adelberger, Test of the equivalence principle using a rotating torsion balance, Phys. Rev. Lett. 100 (2008) 041101 [arXiv: 0712.0607] [INSPIRE].

[39] C.D. Kreisch, F.-Y. Cyr-Racine and O. Doré, The Neutrino Puzzle: Anomalies, Interactions and Cosmological Tensions, arXiv:1902.00534 [INSPIRE].

[40] M. Baryakhtar, R. Lasenby and M. Teo, Black Hole Superradiance Signatures of Ultralight Vectors, Phys. Rev. D 96 (2017) 035019 [arXiv:1704.05081] [INSPIRE].

[41] J. Edsjo, J. Elevant, R. Enberg and C. Niblaeus, Neutrinos from cosmic ray interactions in the Sun, JCAP 06 (2017) 033 [arXiv: 1704.02892] [INSPIRE].

[42] A.M. Dziewonski and D.L. Anderson, Preliminary reference Earth model, Phys. Earth Planet. Inter. 25 (1981) 297. 